\title{
Information Integration of Virtual Enterprise Based on Service-Oriented Architecture
}

\author{
Jun Yang ${ }^{12}$, Gang $\mathrm{Li}^{1}$, and Danxiang $\mathrm{Ai}^{2}$ \\ 1 Wuhan University, School of Information Management, \\ 430072 Wuhan, China \\ imio2@whu.edu.cn, \\ www home page: http://sim. whu.edu.cn \\ 2 Guangdong University of Technology, School of Economics and \\ Management, 510520 Guangzhou, China \\ jgxy@gdut.edu.cn \\ www home page: http://jgxy.gdut.edu.cn/
}

\begin{abstract}
The operation of the virtual enterprise requires the information sharing of the member enterprises. So it is imperative to build up a highquality information integration platform. This paper analyzes the information integration theory of virtual enterprise. It introduces the basic concepts and methods of service-oriented architecture and brings forward the targets of the information integration in the virtual enterprise. Finally, this paper elaborates on the solution of the virtual enterprise information integration based on the service-oriented architecture.
\end{abstract}

\section{Introduction}

Today, we are entering an age when economy is becoming global and advanced technologies are developing rapidly. The competition among the enterprises becomes fiercer. The enterprises are facing a brand-new environment. A new kind of organization-virtual enterprise is coming into being.

However, the base architectures of the member enterprises in the virtual enterprise are different. In many cases, the member enterprises use different communication protocols and data formats. So it is difficult for the members to communicate and integrate information, which decreases the efficiency of the cooperation in the virtual enterprise. How to build up an information integration platform for the virtual enterprise has become a hot issue in recent years. However, much research on the information integration of the virtual enterprise is descriptive. There is little research on the actual organizing of the information integration

Please use the following format when citing this chapter:

Yang, J., Li, G., Ai, D., 2007, in IFlP International Federation for Information Processing, Volume 251, Integration and Innovation Orient to L-Society Volumel, Wang, W. (Eds), (Boston: Springer), pp. 282-239. 
platform of the virtual enterprise. Based on the technologies of service-oriented architecture, this article discusses the information integration framework of the virtual enterprise.

The remaining of this paper is organized as follows. Section 2 gives a brief introduction on the information integration theory of the virtual enterprise. Section 3 discusses the basic knowledge of service- oriented architecture (SOA). Section 4 probes into the information integration model of the virtual enterprise based on SOA. Section 5 is the conclusion.

\section{Virtual enterprise information integration}

\subsection{Virtual enterprise}

It has been showed by Zbigniew Kierzkowski [1] that the virtual enterprise is a kind of dynamic alliance which is built up to grasp special commercial opportunities. It is composed of the core enterprise(s) and partner enterprises. There is probably only one core enterprise or there are probably several core enterprises. The number of the core enterprises depends on the scale of the virtual enterprise. To make things simple, it is supposed in this paper that there is only one core enterprise in the virtual enterprise. The enterprise which has stronger strengths in the industry acts as the core enterprise. And other enterprises in the industry which are appealed to participate in the virtual enterprise act as the partner enterprises. Both the core enterprise and partner enterprises are called the member enterprises of the virtual enterprise.

Through the information technologies, the core enterprise and the partner enterprises establish the temporary alliances to grasp the dynamic opportunities. During the co-operation, the members jointly share the benefits and risks. When the expectant goal is realized, the virtual enterprise will disappear. And when the new commercial opportunities appear, the new virtual enterprise will come forth. It has been showed by Stafford S Cuffe[2] that the greatest difference between the virtual enterprise and the traditional enterprise is that the member enterprises of the virtual enterprise need not to have the complete processes which the traditional enterprises have. What the members in the virtual enterprise should do is remaining and strengthening their core processes and outsourcing their un-core processes.

\subsection{Core information processes of virtual enterprise}

Essentially, the virtual enterprise is the aggregation of the core information processes of different enterprises. There are two kinds of core information processes in the virtual enterprise:

(1) The core information processes of the core enterprise. Usually, the core enterprise of the virtual enterprise is the enterprise which has stronger strengths in the industry. The core enterprise has enough resources to associate the appropriate cooperative partners to build up the virtual enterprise. The core information 
processes of the core enterprise include strategic planning information process, researching and developing information process and so on.

(2) The core information processes of the partner enterprises. For the partner enterprises, their division of labor on the basis of specialization is very clear. And their core information processes are compatible and complementary. The core information processes of the partner enterprises include supplying information process, manufacturing information process, marketing information process, financing information process and so on.

All in all, the core information processes in the virtual enterprise are composed of the core information processes of the core enterprise and partner enterprises. And the core information processes of the core enterprise play a dominant role and can integrate the core information processes of the partner enterprises in the virtual enterprises.

\subsection{Virtual enterprise information integration theory}

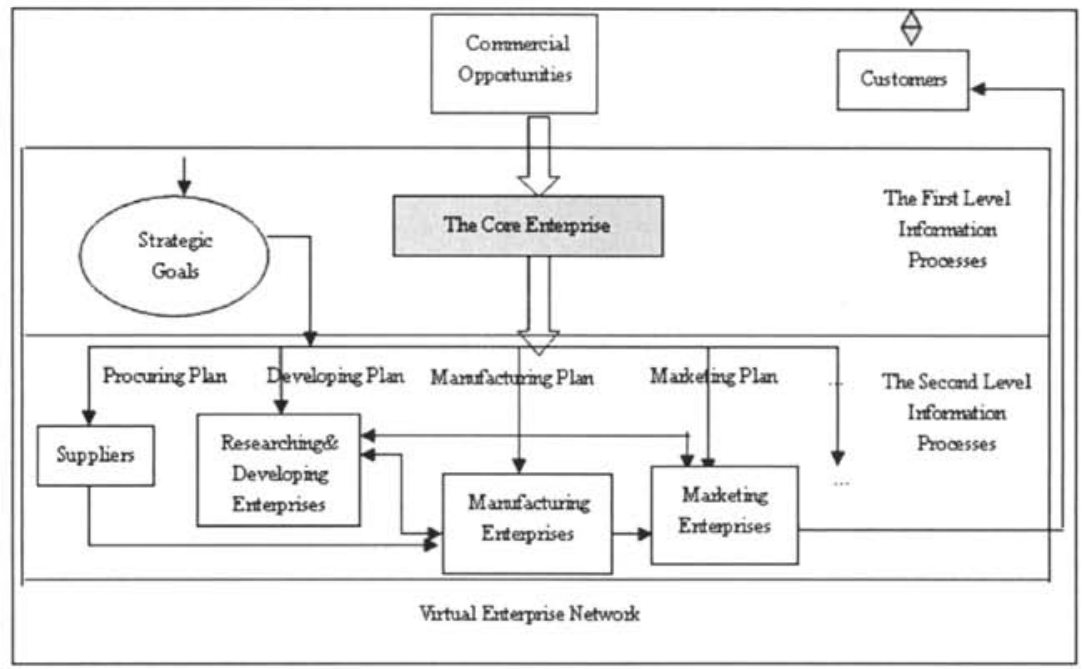

Fig. 1. Information Processes Framework in Virtual Enterprise

It has been showed by Guoqing Huo[3] that the virtual enterprise's lifecycle is following the unceasing information process integration. In the course of the information integration, every member enterprise becomes a subsystem of the whole information system. The core information processes in the virtual enterprise can be divided into two levels: the first level and the second level. The first level information processes indicate the core information processes of the core enterprise. The second level information processes indicate the core information processes of 
the partner enterprises. Every core information process delegates the core ability of a member enterprise. The framework of the information processes in the virtual enterprise is showed in figure 1.

During the course of the establishment and the operation, the virtual enterprise centers on the core information processes of the core enterprise, searching for, optimizing and integrating the core information processes of the other partner enterprises. When the internal, external environment and the core abilities of the member enterprise change, the virtual enterprise will adjust and re-integrate the core information processes of the member enterprises. This integration theory is called virtual enterprise information integration theory.

\section{Service- Oriented Architecture (SOA)}

\subsection{Definition of Service- Oriented Architecture (SOA)}

It has been showed by Jonathan Katz [4] that service means a customer-based or user-oriented function, such as technical support or network provision. Technically speaking, service is a program or routine that provides support to other programs in reference to programming. It has been showed by N Bieberstein [5] that SOA is a kind of groupware model. Several recent investigations $[6,7,8]$ indicate that $\mathrm{SOA}$ connects the services by interfaces and contracts defined in a neutral way which is independent of hardware, operating systems and programming languages. This makes the services can communicate in a uniform mode. Several recent investigations [9-13] indicate that SOA has the following characteristics:

(1) SOA is not a language or a material product. It is a kind of software architecture, which is mainly used to solve the integration problem of the different appliances on the web.

(2) Service is the core of SOA. Service is a kind of application which is encapsulated as business process. And information process is the exterior form of business process. So, it is regarded that service is a kind of application which is encapsulated as information process. In SOA, service is the core element.

(3) SOA is loose-coupling. Coupling means the relationship of different softwares, which includes relativity and dependence between the softwares. The traditional software is close-coupling. It is very difficult for the sub-systems to communicate and to be unpacked. However, in SOA, the software is loose-coupling. The service providers use the uniform definition language to define the interfaces. As long as the interfaces remain unchanged, the internal changes of some applications have no effects on the others.

(4) SOA is mutual-operational. In SOA, the users can use any function on any platform regardless of the programming languages and operating systems. This ensures the solutions based on SOA to be easily integrated. 


\subsection{Structure of SOA system}

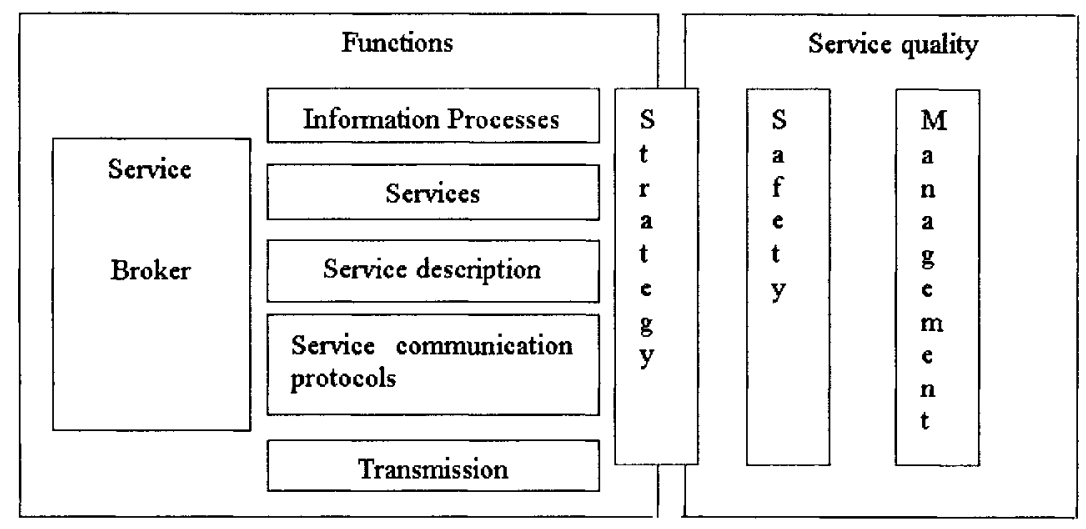

Fig. 2. Structure of SOA System

It has been showed by Wang Jian-xin [14] that SOA is an information integration system based on Internet. Its structure is showed in Figure2. SOA adopts the services-oriented software encapsulation technologies. The main elements of SOA are service-description, service-publishing, service-finding and service-calling. SOA is based on XML (eXtensive Markup Language) and uses Web Services Definition Language (WSDL) to describe interfaces. The service broker is the supporter of service-finding. SOA is not only a software developing framework but also a business developing framework. It can integrate different services on different platforms.

\subsection{SOA and Web Services}

It has been showed by Marshall Breeding [15] that a Web service is a kind of software application which can be identified by URI (Uniform Resource Identifier). Its interface and binding can be defined, described and found as the XML-supported resources. It has been showed by $\mathrm{H} \mathrm{K}$ Cheng [16] that Web Services is an important technology to realize SOA. Web Services supports XML-based messages to communicate with other software agents and can integrate the applications on Intranet, Extranet and Internet. Web Services architecture involves many layered and interrelated technologies. Figure 3 shows how the Web Services technologies match SOA.

Web Services uses Web Services Flow Language (WSFL) to model the information processes, uses Web Services Experience Language (WSXL) to distribute the information processes, uses Universal Description Discovery and Integration (UDDI) to register and find the services, uses Web Services Definition Language (WSDL) to define the services and interfaces, uses Simple Object Access 
Protocol (SOAP) to realize the communication between the service providers and service requesters, uses HTTP, FTP, SMTP to transmit the messages.

From the above analysis, it can be concluded that SOA is not equal to Web Services. SOA is a conception, a methodology and a model. And Web Services is a kind of concrete technology to realize SOA.

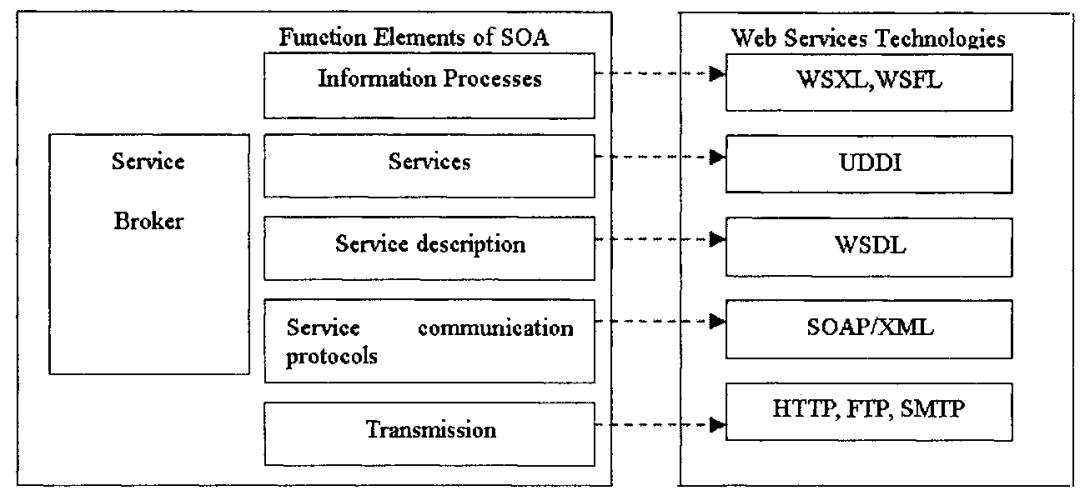

Fig. 3. Matching between Web Services Protocols and SOA

\section{SOA-based virtual enterprise information integration model}

\subsection{Information integration goals in virtual enterprise}

It has been showed by Hamid Haidarian Shahri [17] that the operation of the virtual enterprise requires the member enterprises, which come from different places and have different applications, to be able to share information instantly. So, it is imperative for the virtual enterprise to set down a high-effective information integration scheme. This scheme should realize the following goals:

(1) Reducing the reduplicate resources: It has been showed by Xu Ying [18] that the enterprise should reduce the reduplicate resources in information integration. The core enterprise needs not to offer multiform interfaces to realize the information integration. The scheme should provide a uniform definition language for the interfaces. This can dramatically reduce the reduplicate constructions.

(2) Realizing the loose coupling of integration: Virtual enterprise should make it easy to integrate different information coming from different systems.

(3) Updating the interfaces rapidly: Every member enterprise can realize the corresponding alteration automatically when any other member makes any change in the integration system.

(4) Integrating actively: Every member enterprise can automatically discover and integrate the information of any other member enterprise. 
(5) Being independent of environment: It has been showed by YeYu-Feng Shahri [19] that virtual enterprise should offer an information integration model which can be applied in different systematic platforms and technical architectures.

\subsection{SOA-based virtual enterprise information integration solution}

4.2.1 General framework of SOA-based virtual enterprise information integration model

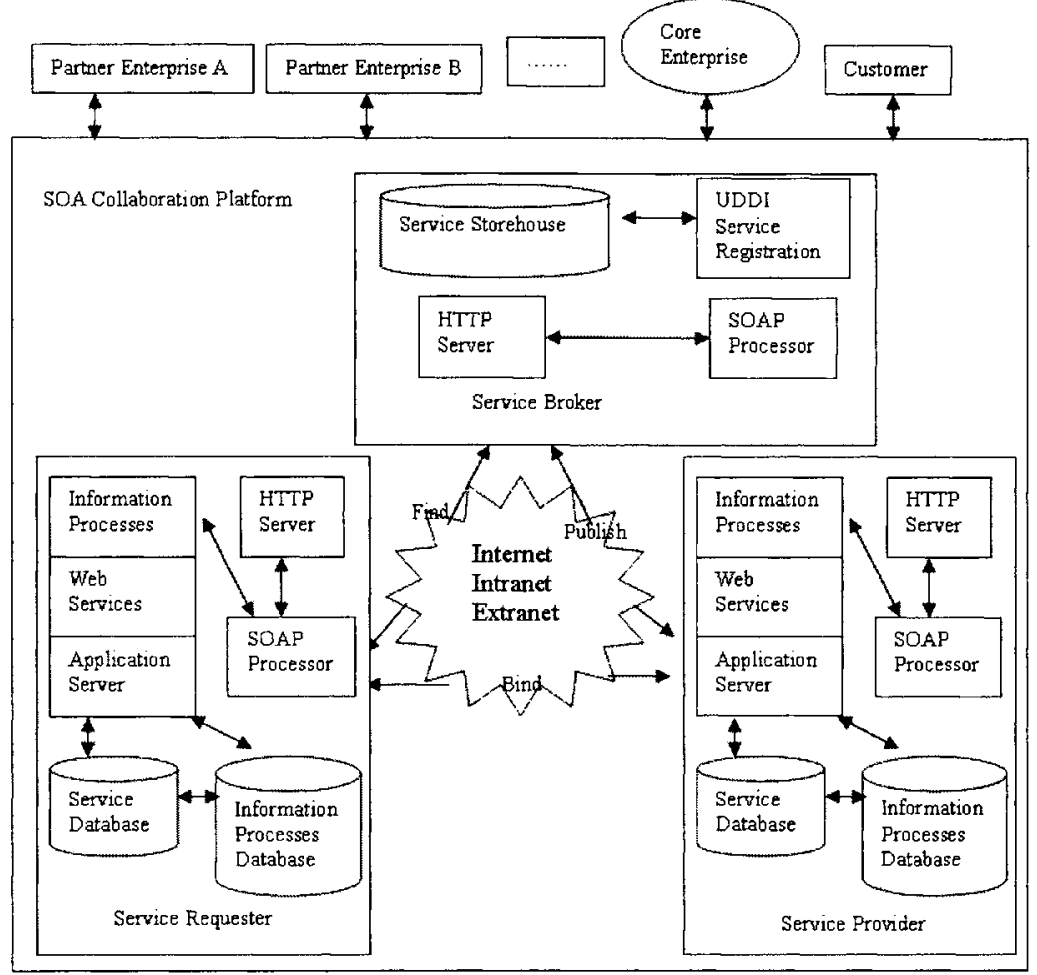

Fig. 4. General Framework of SOA-based Virtual Enterprise Information Integration Model

The member enterprises of the virtual enterprise probably have different development platforms and languages. Several recent investigations $[20,21]$ indicate that the information integration of the virtual enterprise not only requires the members to exchange information effectively but also allows the members to deal with the remote orders and inquires etc. SOA can meet these needs. Using the widely used Web Service technologies, this paper construct an information integration model based on SOA, which is showed in figure4. 
There are three principal roles in figure4: service requester, service provider and service broker. Figure 5 describes the cooperation among the three roles.

(1) Service Broker: It is the supporter of service-finding. It includes a storehouse of the available services. It allows the service requester to search for the interfaces of the service providers. The service broker can receive the finding requirements from the service requesters and the publishing requirements from the service providers, and, return the corresponding responses to the requesters and providers. The service broker receives and returns the messages through HTTP. All these messages are dealt with in the SOAP processor.

(2) Service provider: It is an entity whose IP address can be found. It uses WSDL to describe and define the services and interfaces. It publishes the services by communicating with the service broker and enables the services to be accessed, received and called by the requesters.

(3) Service requester: It searches for the service(s) needed through the service broker, downloads the WSDL file(s) and calls the service(s). So, firstly, the service requester needs to set up the querying mechanism according to the information requirement. The mechanism should offer the querying conditions such as querying according to interface type and so on. Secondly, the querying conditions should be encapsulated as a SOAP request message by SOAP development tools and sent to the service broker. After dealing with the querying, the service broker encapsulates the querying results as a SOAP response message and returns it to the service requester. After receiving the response message, the service requester calls the service(s) through SOAP. Finally, the called service(s) encapsulates the serviceresult(s) in a SOAP message and returns it to the service requester.

In the virtual enterprise, it is not fixedly regulated whether the core enterprise or ordinary partner enterprises should act as the service requester or the provider. It should be flexibly decided according to the actual conditions. In the anterior content, we have discussed that the information integration of the virtual enterprise is the complementary integration of the core information processes of the member enterprises. So when an information process is called, the enterprise (the core enterprise or ordinary partner enterprise) who offers this information process is the service provider. And the enterprise (the core enterprise or ordinary partner enterprise) who calls this information process is the service requester. 


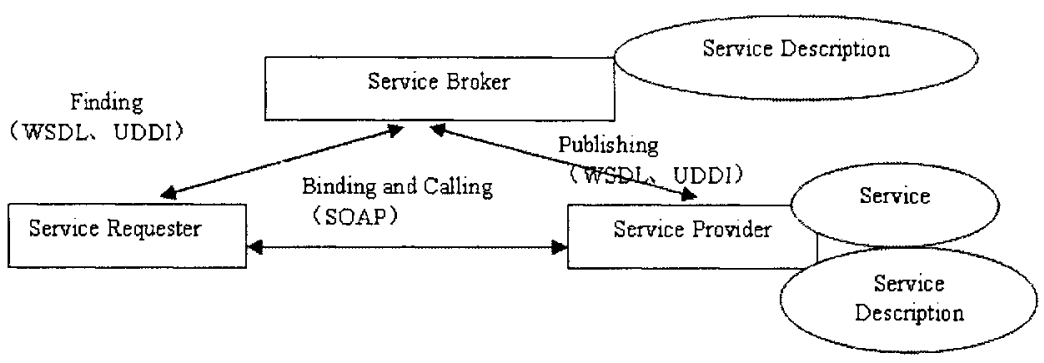

Fig. 5. The Cooperation in SOA

4.2.2 Detailed analysis of SOA-based virtual enterprise information integration solution

In the above content, we adopt Web Services technologies to construct the general framework of SOA-based virtual enterprise information integration model. This framework can realize the automatic information integration among the member enterprises. How the core enterprise, partner enterprises and customers build up the SOA platform and integrate information will be described in details in the following content. The status and functions of the core enterprise, partner enterprises and customer are showed in figure6. Figure 6 is drawn on the base of figure5. So, in order to make figure 6 be easily analyzed, the same parts as Figure 5 such as the SOAP Processor and HTTP Processor are omitted. 


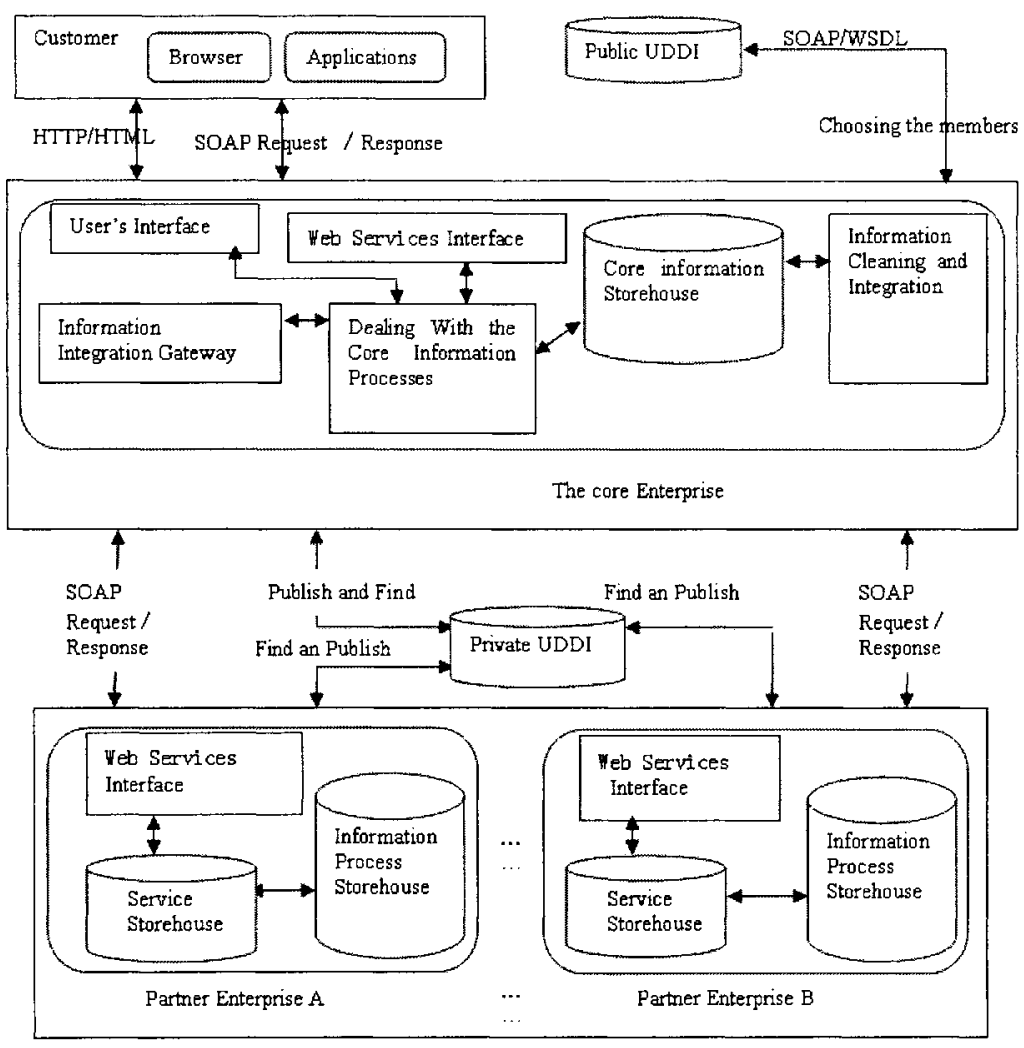

Fig. 6. Detailed Framework of SOA-based Virtual Enterprise Information Integration Model

(1) Core enterprise

The framework is built up centered around the core enterprise which leads all the members to actively establish the SOA platform. After inquiring about the information of the registered enterprises in the public UDDI, the core enterprise chooses the compatible members. The core enterprise needs to set up and maintain the private UDDI which is provided only to the members to guarantee the security and the validity of the information. The internal system of the core enterprise should be able to clean and integrate the information, so that the information read from the Web Services interfaces could be filtered before it entering the storehouse. The core enterprise should provide the customers with web-based user interfaces. And the core enterprise should analyze the request messages from the customers and send them to appropriate partner enterprises.

(2) Partner enterprises

The partner enterprises publish the interfaces of the information process applications in the form of Web Services. According to their own needs, the partner enterprises make decisions on whether they should register the services in the private 
UDDI or directly integrate the services into the core enterprise. The partner enterprises build up a layer of special information exchange module between their internal system and the external Web Services in order to realize the fluent information exchange. The internal core information processes of the partner enterprises are stored in the information process storehouse. The partner enterprises should be able to offer the outsourcing and integration services of different information processes to other partner enterprises and customers.

(3) Customers

The customers communicate with the core enterprise through the web browsers or applications. When the browsers are used, the communication happens in a universal electronic commercial mode. When the applications are used, the communication happens in the way of calling Web Services.

The information integration of the virtual enterprise is centered on the core information processes of the core enterprise, along with the continuous search, optimization and integration of the core information processes of the complementary partner enterprises. From figure 6 , it can be concluded that both the core enterprise and partner enterprises can be the service requesters finding the information services and the service providers publishing the information services.

\section{Conclusion}

How to realize the effective information integration of the virtual enterprise has been a difficult problem and challenge for many enterprises. The framework of SOAbased virtual enterprise information integration model solves the information communication and integration problem and makes full use of the existing core information processes of the member enterprises in the virtual enterprise. With the development of the related technologies, SOA will bring great opportunities to the implement and operation of the virtual enterprise. However, there are still many things to do to realize the information integration of the virtual enterprise, for example, the standard of secure message transmitting and Web affair processing should be improved. And these are our future research.

\section{Acknowledgement}

This research was supported by the grand research fund of State's Key research base of humanities and social sciences of China under Grant 05JJD870159.

\section{References}

1. K. Zbigniew, Towards virtual enterprises, Human Factors and Ergonomics in Manufacturing , 15(1), 49(2005).

2. S.C Stafford, Future Virtual Enterprise Strategies For the New Global Economy, Futurics,29(1/2), 46-50 (2005) 
3. G.Q. Huo, Theory and Cases of the Integrated Management Strategies of Enterprise Information Resources, Tsinghua University Press, Beijing(2004).

4. K. Jonathan, SOA: The Next Disruptive Force, Industry Week, 256(3), 41(2007).

5. N.Bieberstein, S.Bose, L.Walker and A.Lynch, Impact of service-oriented architecture on enterprise systems, organizational structures, and individuals, IBM Systems Journal, 44(4), 691-709(2006).

6. D.E. Cox and H. Kreger, Management of the service-oriented-architecture life cycle, IBM Systems Journal, 44(4), 709-726(2005).

7. S. Stephanie, SOA Is Happening-Time To Get On Board, InformationWeek, Feb (1127), 58(2007).

8. K. Eric, "SOA", InfoWorld, 9(1), 16-17(2007).

9. K. Eric and G.Gale, SOA: UNDER CONSTRUCTION, InfoWorld, 28(45),25-31(2006).

10. Charles B., SOA WORK IN PROGRESS, InformationWeek Manhasset,Oct 31(1062), 40-44(2006).

11. David M., SOA PLANNING: Sizing Up Your Business Processes, InfoWorld. Mar 28(11), 18-23(2006).

12. W. Mark, SOA: Enabler of Mass Customization, Computerworld, 40(47), 30(2006).

13. D. Frank, SOA evolves with information services, Network World, 23(42),35(2006).

14. Wang J.X., Lv X.h., Service-Oriented Information Integration Based on Web Services, Computer Era.2, 1-2(2006).

15. Marshall B., Introduction to Web Services, Library Technology Reports.May/Jun 42(3), 5-18(2006).

16. H.K. Cheng, Q.C. Tang and J.L. Zhao, Web Services and Service-Oriented Application Provisioning: An Analytical Study of Application Service Strategies, IEEE Transactions on Engineering Management, 53(4), 520(2006).

17. H. S.Hamid and H. S. Saied, Eliminating Duplicates in Information Integration: An Adaptive, Extensible Framework, IEEE Intelligent Systems, 21(5), 63(2006).

18. Y. Xu an F.Y. Xu, Research on the Supply Network Model Based on SOA, Science and Technology Progress and Policy. 3, 162-164(2006).

19. Y.F. Ye, "Enterprise Application Integration Scheme Based on SOA", Microelectronics and Computer, 23(5), 211-213(2006).

20. H. Ma and J.H. Li, Application of service-oriented architecture in dynamic enterprise application integration, Computer Engineering and Design, 27(13), 2507-2509(2006).

21. S.L. Yang, Y. Liu and X.J. Ma, Virtual Enterprise Solution Based on Web Services and Service-Oriented Architecture, Application Research of Computers, 10, 36-38(2006). 